\title{
Nicolas Venette (1633-1698) : premier sexologue français et grand pionnier en matière de lithiase urinaire
}

\author{
Georges ANDROUTSOS
}

Histoire de la Médecine, Faculté de Médecine, Université d’loannina, Grèce

\section{RÉSUME}

Nicolas Venette (Charles Patin) fut un grand médecin du $17^{\mathrm{e}}$ siècle. II a laissé des ouvrages sur le scorbut, les eaux minérales, la lithiase urinaire, la sexologie, etc. Ses œuvres maîtresses furent « La Génération de l'homme ou Tableau de l'amour conjugal » et le " Traité des pierres ". II est considéré comme le premier sexologue français et un grand pionnier en matière de lithiase urinaire

Mots clès : Venette, pionnier, sexologie, lithiase urinaire

\section{VIE-CARRIERE}

II naquit à La Rochelle. Son vrai nom était Charles Patin. Fils du célèbre doyen de la Faculté de Paris Guy Patin (1600-1672), il écrivit sous le pseudonyme de « Nicolas Venette de la Rochelle » ou « Solocinus le Vénitien » [2].

II fut un réformé si honnête qu'on l'élut doyen des agrégés du Collège Royal de La Rochelle. II exerça la médecine sous le règne de Louis XIV (1638-1715). II s'exila volontairement en Italie et finit par obtenir une chaire à Padoue [6], où il illustra la médecine pratique. II fut aussi célèbre comme numismate [4]. II mourut à La Rochelle.

\section{SON CEUVRE MEDICALE}

Nicolas Venette, installé à La Rochelle, s'était mis à écrire toutes sortes d'ouvrages consacrés au scorbut (« Traité du scorbut et de toutes les maladies qui arrivent sur mer "1671), aux eaux minérales, aux fièvres intermittentes et même à l'art de tailler les arbres! Mais aucun de ces livres ne lui eût valu de passer à la postérité s'il n'avait publié, en 1675, d'abord sous un pseudonyme, son ouvrage capital " La Génération de l'homme ou Tableau de l'amour conjugal " dont la deuxième édition parut à Amsterdam en 1685 et connut un grand succès [7]. II s'agissait pratiquement du premier livre de sexologie qui devait être traduit en plusieurs langues et réédité jusqu'en 1812 une cinquantaine de fois. A juste titre donc Venette est considéré comme le premier sexologue français [3].

Peut-être ne connaît-on pas suffisamment son ouvrage posthume "Le Traité des pierres " [1] (paru à Amsterdam en 1701, trois ans après la mort de son auteur). Atteint dès l'âge de 30 ans de quatre crises de colique néphrétique en une seule année, Venette s'était, toute sa vie durant, appliqué à étudier les causes, les symptômes et aussi les traitements tant prophylactique que curatif de la lithiase rénale.

\section{SON CEUVRE « LA GENERATION DE L'HOMME... »}

\section{Généralités sexologiques}

L'ouvrage " La Génération de l'homme, ou Tableau de l'amour conjugal " [9], contient une préface où se manifeste de façon exemplaire le contexte moral de l'époque. II s'agit d'un ouvrage assez libertin, il accepte l'habitude phénicienne de faire déflorer les filles par les

Correspondance :

Dr Georges ANDROUTSOS - 1 rue Ipeirou, 10433, Athènes, Grèce - Fax +302108235710 - Email lyon48@otent.gr 
valets ou la coutume arménienne de les faire déflorer dans le temple d'Anataïs. II conseille un examen prénuptial sérieux, donne une formule de contraceptif, décrit les grossesses dans les cornes de la matrice, où il pense que se fait la rencontre des semences de l'homme et de la femme ; quant à préparer un garçon, mieux vaux attendre " un souffle de vent du Nord au plein de la lune ". II conclut que les « ressemblances que nous avons avec nos ancêtres sont en puissance dans notre semence " [10].

Venette se montre un défenseur acharné du moralisme chrétien. II réfute tout ce qui s'opposerait à la conception. Il précise que « le poil commence à poindre aux parties naturelles (des filles) vers 12-15 ans... quand l'âme peut distinguer le vice de la vertu ». II demeure persuadé qu'un athée, lisant son livre, « y trouvera de quoi changer de sentiment, reconnaissant clairement Dieu dans le mécanisme de la génération des hommes ". Venette est plus réaliste et se moque du vieil aristotélicisme d'Ambroise Paré, partisan du retournement interne, dans le corps de la femme, des organes génitaux masculins, qui croit que la matrice est « comme le scrotum de l'homme ", son col ressemble à la tête de la verge, et le clitoris - du moins "les nymphes " - se dressent comme la verge virile : " quelle proportion y a-t-il entre la matrice et le gland, entre le membre viril et le clitoris »? ...

La physiologie reproductrice paraît encore bien mystérieuse. Venette demeure hippocratique, ce qui le conduit à recommander l' «émission " orgasmique féminine. La fonction érotique féminine demeure écartelée entre la matrice et le clitoris. L'utérus est toujours le très avide " aspirateur à sperme " responsable des appétits sexuels des femmes. La matrice d'une femme est du nombre des choses insatiables dont parle l'Ecriture, constate Venette. II donne au col de la matrice le nom « d'étui viril ». II le reconnaît pourtant « comme un lieu où les trésors de la nature sont cachés, situé au plus bas du ventre, entre la vessie et le gros boyau qui servent comme de coussins au plus fier et au plus superbe de tous les animaux pendant qu'il demeure dans les flancs de sa mère » [5].

En ce qui concerne le clitoris, « c'est là, écrit le médecin, que la nature a placé ces chatouillements excessifs, et qu'elle a établi le lieu de la lascivité des femmes. Car, dans l'action de l'amour, le clitoris se remplit d'esprit, et se raidit ensuite comme la verge de l'homme. II ne lui manque ni gland, ni prépuce; et s'il était troué par le bout, on dirait qu'il est tout semblable à un membre viril ».

Venette n'a pas compris ce qu'on enlève aux filles
d'Afrique. Mais il regrette que les femmes françaises affligées de nymphes trop pendantes sont trop honteuses pour les exposer au chirurgien qui pourrait les raboter. II fait un tableau horrifiant de l'hypertrophie clitoridienne, monstruosité ne se révélant parfois qu'à la saison chaude, mais pouvant atteindre jusqu'aux reines ! II se résigne à préconiser l'abstention chirurgicale : les tentatives de ligature, ou d'ablation exposent à de fâcheux accidents, qui peuvent mettre en péril non seulement la vie de la malade, mais aussi la réputation du praticien.

Venette n'hésite pas à exalter la beauté des organes de l'homme qui servent à la génération : « Dieu les a créés avec un soin particulier. À voir leur assemblage, leur proportion, leur figure et leur action ; à considérer les esprits qui y sont portés, les chatouillements et les plaisirs que l'on y ressent, l'âme même qui y réside, puisque c'est par là qu'elle sort pour se communiquer à l'enfant, il n'y a point d'homme qui ne les admire et n'y doive faire de particulières réflexions ».

C'est pourquoi, souligne Venette, «quand une femme aperçoit par hasard une verge, par le défaut de quelques replis, elle sent son cœur échauffé au même instant par une passion de laquelle elle ne peut se défendre qu'avec peine». C'est par un mouvement précipité qu'elle s'approche du membre de l'homme, pour en tirer de quoi s'humecter et se procurer du plaisir, «arrachant la semence aussi vivement qu'un estomac affamé arrache la viande de la bouche ».

« La semence de l'homme, écrit encore Venette, se communique à celle de la femme, comme l'humeur de la terre se filtre dans la semence d'une plante par son germe ".

Mais sa saveur est beaucoup plus délicate que la semence de la femme, comme il l'explique plus loin après une dessection faite sur une jeune fille de vingt ans : " Je trouvais, écrit-il, ses testicules si pleins de semence, qu'au premier coup de scalpel, la matière renfermée rejaillit aussitôt sur mon visage, et m'en étant par hasard tombé sur les lèvres, j'y portais la langue sans y penser, et j'en goûtais assez pour la trouver fade, dégoûtante, et un peu austère. Alors que le sperme de l'homme est un élixir délicat " .

\section{Troubles de l'érection et de l'éjaculation}

Venette se montre un grand pionnier en matière de pathologie sexuelle ; la pathologie masculine tient le devant de la scène dans son ouvrage. Les troubles de l'érection paraissent bien compréhensibles à Venette. L'homme se fatigue plus que la femme. C'est lui qui, dans les rapports conjugaux, " agit presque tout seul, tandis que la femme ne fait que souffrir les caresses d'un 
homme dans une posture aisée ». Venette nous gratifie d'un tableau impressionnant de ce qu'il appelle le " dyspneumatisme ". Il est ainsi quasi le seul à dépeindre les troubles de l'éjaculation. Avec pas moins de sept différents cas parmi lesquels, se trouvent l'éjaculation molle et l'éjaculation rétrograde. Les causes de ces embarrassements masculins relèvent de trois grandes entités d'autrefois :

- La morale : On y trouve ressassées les vieilles rengaines sur l'épuisement par la vie dissipée, les excès vénériens, l'abus du plaisir solitaire... Dédouanement moralisateur auquel Venette joint le très étrange " saignement pseudomenstruel ".

- Les sortilèges : Venette a compris le mécanisme des entraves fonctionnelles liées à la crainte de l'échec, en particulier chez les esprits faibles. II ne croit pas qu'un magicien puisse s'opposer aux violents desseins de la nature: « les belles portent avec elles un philtre et un sortilège bien plus puissants, et c'est contre celui-ci qu'il y a peu de remède ".

Il confirme de façon simple sa théorie sur l'inquiétude des faibles de caractère. Un tonnelier employé de son père aurait dit du mal de lui. Venette menace de lui nouer l'aiguillette et de fait, marié peu de temps après le tonnelier reste coi plus d'un mois auprès de sa jeune épouse. II parvient heureusement à se faire désempêtrer en trois semaines par son curé... après quoi il peut faire des enfants [10].

Venette traite de superstitions les fameux contrecharmes délivrant l'aiguillette nouée. " L'ellébore et le vin émetique " sont plus efficaces, et il vaux mieux purger les sorciers, illuminés ou charlatans, que les brûler.

- Les causes organiques : Venette connaît les imperfections anatomiques masculines, l'hypospadias, le phimosis, la brièveté du frein, ainsi que le paraphimosis, "renversement du prépuce ", accident banal du dépucelage. II dispute des dimensions fautives du membre viril, trop court, ou trop long, ou trop large. Les modèles grande taille ne lui paraissent pas les meilleurs. II accuse les gens aux grandes verges d'être « les plus étourdis et les plus stupides des hommes ".

La classification des causes d'impuissance organique, qui reste encore d'actualité, fait l'honneur de Venette et mérite d'être présentée :

a) Les causes endocriniennes : insuffisance ou absence des testicules. Venette sait que l'ectopie, cause certaine de stérilité, n'est pas cause d'impuissance si l'homme possède vigueur, pilosité et voix grave [8]. b) Les causes traumatiques : l'homme a reçu un grand coup (qui a pu ébahir les nerfs érecteurs).

c) Les causes infectieuses : une humeur en abondance (qui a provoqué une prostatite chronique).

d) Les causes toxiques: l'alcoolisme.

e) Les causes neurologiques : les nerfs qui sortent de l'os sacrum sont " faibles, ou bouchés "; ou l'homme souffre d'une paralysie des membres inférieurs.

f) La sénescence : l'atteinte athéromateuse des artères.

g) Les nodosités : il s'agit de la maladie de Lapeyronie dite " induration plastique des corps caverneux ", responsable de déviations de la verge.

h) Les freins trop courts : Venette propose une section transversale et préconise l'hémostase au blanc d'œufs battu. II recommande un geste chirurgical plus correct que l'incision « artisanale » pratiquée par les matrones italiennes qui agissaient grâce à l'ongle, conservé long, de leur pouce droit...

i) Le phimosis : Venette propose une opération " par des incisions de chirurgie plastique ", comme par les Antiques, et non par la circoncision judéo-islamique. II insiste sur la "bienséance " de conserver une couverture au gland. II préconise même de « reconstituer un prépuce » aux Juifs, Turcs et autres circoncis qui en feraient la demande. II préconise une incision circulaire derrière la couronne du gland, permettant de le recalloter progressivement.

Venette connaît les aphrodisiaques mais ne leur accorde pas grand crédit. II met en garde contre leurs dangers, en particulier la néphrite hématurique mortelle provoquée par la cantharide. De même qu'il compte sur le charme naturel des femmes pour combattre les maléfices de sorciers, il s'en remet à la plus classique - pour nous - thérapeutique de couple en matière d'impuissance. Avec un réalisme amer : " si la main d'une belle femme, qui est le plus excellent de tous les remèdes, n'a pas assez de vertu pour guérir la molesse de la verge d'un homme, les autres remèdes y auront peu de force ".

\section{Malformations péniennes}

Venette reconnaît honnêtement que l'homme n'est pas, lui non plus, dénué d'infirmités. Il s'attarde sur les verges trop courtes ou trop longues, d'autant que ces dernières ont la réputation d'être stériles, la semence se dissipant dans le conduit d'une longue verge.

Venette ne croit pas cependant que ces verges démesurées soient stériles, " car la semence se porte directement au fond de la matrice, à moins qu'étant peu maniables, elles ne rendent impossible le coït ». 
"Les hommes qui ont de longues verges ont aussi un grand nez, et ils sont beaucoup plus robustes et courageux que les autres. Nous ne devons pas nous étonner de ce qu'Héliogabale, que la nature avait favorisé de grandes parties génitales, choisissait des soldats qui avaient de grands nez. Mais il ne se doutait pas en même temps que ces gens aux grandes verges étaient les plus stupides des hommes $"$.

Pour les verges trop longues, il préconise l'usage d'un écusson de cuir, de liège bien cotonné, renouvelé des anneaux antiques, pour ne pas " empaler " le cul-desac postérieur de leurs partenaires.

En ce qui concerne les verges trop courtes, Venette dénonce les remèdes de bonne femme censés les allonger, frictions, onguents, emplâtres, voire la boule qui perce le prépuce et à laquelle on suspend une balle de plomb...

\section{Contre les superstitions}

Par rapport aux femmes, Venette rejette l'idée qu'elles puissent être engrossées en se baignant dans une étuve publique, comme essaient de le faire croire certaines coquines « pour couvrir leur lascivité et cacher le vice d'un amour impur ». II ne croit pas non plus aux contes des incubes et des succubes accusés d'engrosser les femmes, et d'ailleurs, ils en seraient bien incapables, "car que peut-on espérer d'une semence éventée et froide qui aura touché un cadavre, d'un corps d'air que le démon aura emprunté ? "

Venette pense d'ailleurs que la croyance des femmes en l'existence des incubes, vient d'une trop longue continence, qui provoque des suffocations nocturnes. Une femme mélancolique « s'imagine aisément qu'elle est la proie d'incubes lubriques ». Parfois, suggère-t-il, le succube n'est qu'une tribade qui l'embrasse étroitement et en jouit au lieu du démon dont l'autre pense être amoureusement caressée. De même, il juge impies les expériences de certains savants pour créer des bébés artificiels en mélangeant du sperme avec du sang menstruel, ou en introduisant par artifice de la semence dans la matrice de la femelle. "Ce ne sont que sornettes que tout cela ", écrit-il, sans prévoir que ces exploits seront réalisés plus tard par le savant Lazzaro Spallanzani.

\section{Comment faire l'amour pour procréer}

Avant tout, la femme doit danser, "car les femmes s'étant agitées avant de se joindre amoureusement à leur mari, sont défaites d'une partie de leurs excréments, et la chaleur qu'elles ont acquise en dansant a servi à dessécher leurs parties amoureuses, qui ne sont le plus souvent que trop humides, et qui, par ce moyen, ne sont pas disposées à la génération, car la trop grande humidité de ces parties est une des principales causes de stérilité ».

De son côté, le mari n'entrera pas dans le lit conjugal " avant que le ventre et la vessie n'aient rendu leurs excréments, autrement le plaisir y sera bien petit, et l'effet que l'on souhaite quasi inutile, parce que le sperme ne peut être expulsé librement quand la vessie est pleine d'urine, ou le boyau droit empli de matière fécale ".

Tandis que le mari défèque, l'épouse prépare une fumigation d'herbes chaudes cuites dans du bon vin, pour en imprégner ses parties génitales, et introduit délicatement dans le col de sa matrice un peu de musc et de civette.

«Et lorsqu'elle se sentira aiguillonnée et émue, elle le dira à son mari, et alors, se joindront ensemble, et accompliront leur jeu doucement ».

Les différentes positions coïtales ne semblent préoccuper que Venette. Dans quelle position les époux accompliront-ils l'œuvre de chair ? II en existe quatre : debout, assis, face à face et par derrière. II critique les postures debout ou assises, qui procurent une " mauvaise aspersion " de la matrice, et la posture d'Andromaque, qui brave l'empire que I'homme doit avoir sur la femme.

II explique : "Nos parties amoureuses ne sont pas faites pour nous caresser debout comme des hérissons. Cette posture altère notre santé, provoque des éblouissements et des maux de tête, des tremblements de genoux. C'est une source de rhumatisme et de goutte ". En outre, les enfants qui naîtront risquent d'être difformes.

Venette condamne ensuite l'amour assis, " parce que les parties naturelles ne se joignent qu'avec peine, et que la semence n'est pas reçue parfaitement ". C'est la femme qui, le plus souvent, inspire à l'homme cette posture impudique, et Venette blâme l'homme qui s'y soumet, car " l'amour assis rend stérile, ou bien les enfants qui en naissent sont nains, boiteux, bossus et stupides ».

Venette approuve l'amour couché face à face, ou même de côté, surtout si l'homme, trop pesant, risque de provoquer des fausses couches. Mais il hésite avant de se prononcer sur l'amour par derrière, "cette posture étant contre les lois de la nature ".

« Pourtant, consent Venette, toutes les bêtes se joignent de la sorte, et la matrice de la femelle est alors plus apte à recevoir la semence du mâle; elle la retient et la fomente plus commodément lorsqu'une femme est sur les mains et les pieds, que lorsqu'elle est sur le dos».

Quoique Venette n'encourage pas entièrement cette 
position hétérodoxe, il reconnait qu'elle est utile lorsque l'homme trouve des obstacles qui empêchent d'embrasser sa femme par devant. Par exemple « un ventre en pointe " qui s'oppose à l'approche du mari. Venette va jusqu'à citer saint Thomas d'Aquin qui conseillait aux hommes trop gros de prendre leurs femmes par derrière, surtout vers la fin de la grossesse pour ne pas risquer d'étouffer l'enfant.

Quelle que soit la position choisie, Venette conseille de ne pas abuser du coït si l'on souhaite avoir des garçons plutôt que des filles, et de ne pas se caresser plus de trois ou quatre fois par mois, pour conserver dans la semence les " esprits " nécessaires à la fabrication des mâles.

Quant à la stérilité, la femme seule en est responsable parce qu'elle est sujette à beaucoup plus d'infirmités que l'homme. La stérilité vient le plus souvent de son côté. Elle peut provenir « d'un conduit de la pudeur trop étroit...». De toute manière, tous les vices et irrégularités de la conception viennent de l'épouse.

\section{SON CEUVRE « LE TRAITE DES PIERRES »}

Son ouvrage « Traité des pierres qui s'engendrent dans les Terres et les Animaux, où l'on parle exactement des causes qui les forment dans les Hommes, avec la Méthode de les prévenir et les abus pour s'en garantir et les chasser même hors du corps " contient le résumé de ses recherches sur cette maladie " une des plus sanglantes et épineuses qui se trouvent dans la médecine " d'après la préface des éditeurs hollandais. Certes, ce n'est qu'un document bien désuet à beaucoup d'égards, mais, compte tenu de l'époque et de l'état des connaissances scientifiques du siècle, cet ouvrage témoigne des dons d'observateur et de l'esprit clinique de son auteur dont on ne saurait nier les grandes qualités professionnelles.

" La Médecine, dit-il, n'a point de meilleurs raisonnements que ceux qui se confirment par plusieurs expériences " et ce n'est point la faute de cet adepte de la méthode expérimentale, si la physique de son temps ne lui apporte pas les lumières dont il aurait besoin pour étudier les pierres et les mystères de la nature. Frappé par les divergences d'opinions des anciens auteurs sur l'origine des pierres que l'on trouve dans le corps humain il est convaincu que le phénomène si particulier de la pétrification que l'on observe autour de certaines sources thermales est l'explication de la production in vivo de la gravelle. II pense donc que celle-ci résulte d'un « esprit fermentatif pétrifiant " et c'est précisément parce que « cet esprit coagulant qui fait les pierres comme l'esprit de vin fait le caillé " produit l'admirable ouvrage de la pétrification autour de certaines sources que celles-ci sont particulièrement nocives dans le traitement des pierres.

II n'en rapporte pas moins les différentes théories pathogéniques de cette maladie qu'il « considère comme le plus grand de tous les maux ". Tandis que "Galien, Jules Scaliger et Albert le Grand pensent que les pierres se forment par une faculté, une propriété, une vertu ou une force lapidifiant " et que Paracelse incrimine la formation d'une espèce de tartre dans nos veines « parmi nos liqueurs ", Fernel - l'un des plus grands médecins de ces derniers siècles, au dire de Venette - fait intervenir la complexion naturelle pierreuse et sablonneuse de la substance des reins, venue du tempérament des reins des parents. Il est évident que cette notion diathésique, pourtant si importante, a complètement échappé à Venette, mais on doit rendre hommage surtout à ses talents de clinicien avisé, d'hygiéniste perspicace et de thérapeute prudent.

Ayant poursuivi ses études médicales « tant en France qu'en Italie, en Suisse et au Portugal " ainsi qu'il le rappelle dans son ouvrage, Venette avait évidemment beaucoup vu et beaucoup retenu. Son expérience clinique s'était considérablement enrichie jusqu'au jour où il colligea le fruit de ses observations dans cet ouvrage qui fut le couronnement de sa carrière. II avait aussi compulsé tous les auteurs anciens ayant traité de la question.

Personnellement, il rappelle qu'il avait trouvé au cours de ses dissections "dans le théâtre anatomique de Padoue un homme mort hectique à l'Hôpital qui avait un rein tout puruleux et l'autre rempli de quatre pierres renfermées dans la membrane du rein sans chair ". Trente ans plus tard, en mai 1692, Venette assiste à une opération de la taille pratiquée par un lithotomiste d'Aunis, le sieur Bourneuf, sur l'enfant d'un boucher de la Rochelle qui était porteur d' "une pierre grosse comme le pouce, d'une couleur cendrée et parsemée de gros sablons rouges ». Néanmoins, le clinicien est frappé par la rareté de la gravelle dans la population des îles de Ré et d'Oléron. II prétend même que « la plupart des paysans des costes de cette Province qui ne vivent presque de coquillages, avec du pain, ne sont pas si sujets à la pierre que les autres et - dit-il - nous n'avons pas observé jusques icy qu'entre dix mille de ces personnes il y en eust plus d'une ou deux de maltraitées par la pierre ". II est évident que la frugalité alimentaire des milieux ruraux d'alors pouvait expliquer cette immunité relative de la population autochtone de l'Aunis, alors que la noblesse et la bourgeoisie payaient, du fait de leurs excès alimentaires et aussi de leur sédentarité, un lourd tribut au deux grandes maladies de la nutrition, " la goutte et la gravelle, ces deux sœurs ", comme 
écrivait Erasme à son ami de Londres Thomas Morus.

Venette met en garde, en effet, contre les dangers de l'immobilité prolongée « les personnes déjà âgées qui sacrifient toute leur vie à la contemplation de la nature et à la lecture des archives de l'Antiquité, qui remportent pour gage de leur travail, une pierre dans le rein ou dans la vessie ". Telle est la triste rançon que payent, au terme de leur vie, les hommes d'étude qui, suivant la pittoresque expression du doyen rochelais «se bâtissent souvent un tombeau de pierre dans leur propre corps avant que de mourir ». Chez tous ces ralentis de la nutrition, Venette signale fort pertinemment l'extrême fréquence " aux doigts des pieds et des mains des pierres tendres » alors désignées en France sous le nom de « tuf » c'est-à-dire nos tophi actuels. II rappelle aussi, après Jean Wier et Cordon, la présence dans les urines « de fils comme de la laine, blancs, noirs, jaunes, longs et forts, quelquefois doubles ou nouez " qu'une demoiselle malade de la pierre gardait « dans une boëte " en souvenir de sa cystite.

Ainsi les faits cliniques intéressants ne manquent point dans ce traité où est naturellement signalée la fréquente concomitance des lithiases rénale et biliaire, ainsi qu'il résulte de l'autopsie de Jean Chataigner, seigneur de la Rocheposay, dont la vessie contenait une grosse pierre couverte de glaires, plusieurs dans la vessie et une petite dans la vésicule du fiel, ovale, inégale et noire. C'était le moment où à Bologne, le prince des anatomistes italiens, Malpighi, faisait ses plus retentissantes découvertes, mais on voit que, en France, l'anatomie passionnait aussi les chercheurs et ce n'est qu'au siècle suivant que Morgagni insistait sur la coïncidence de la gravelle biliaire et de la gravelle urinaire.

Néanmoins, c'est surtout en matière d'hygiène et de diététique que Venette affirme sa maîtrise et révèle toute l'acuité de son bon sens. II met naturellement ses patients graveleux en garde contre tous « les excès que l'on fait dans le manger et dans le boire qui remplissent nos corps d'ordures, minent le tempérament de nostre estomach et de nos autres-parties et rendent faible tut notre corps ». N'abusons point du sommeil et du repos qui doivent être "médiocres " et au lieu de nous coucher sur le dos dans un lit de plumes, dormons en décubitus latéral sur un matelas dur. Gardons-nous bien de résister longtemps au besoin d'uriner, car c'est alors que " l'urine fait des concrétions pierreuses qui s'endurcissent avec le temps et y forment la pierre ». Il ne suffit pas d'ailleurs de combattre toutes les causes de rétention d'urine, il faut aussi laver l'arbre urinaire et, pour pratiquer largement la cure de diurèse, l'auteur conseille l'ingestion de boissons très abondantes ; froides l'été et chaudes l'hiver.

Contrairement à la majorité des médecins de son époque, Venette considère que le lait, fort aisé à digérer, est une excellente boisson pour les graveleux et qu'il ne prédispose nullement à la lithiase, de même d'ailleurs que le petit lait qui, précise-t-il, " s'oppose à la pierre ". Autant il proscrit le vin rouge, " gros, épais, terrestre et astringent » autant il recommande, le vin blanc en paillet, qui a une couleur d'œil de perdrix, qui pétille dans le verre, qui est bien " meur », d'un an et tiré d'un tonneau plein, qui est d'une substance tenue et légère. Le cidre est aussi permis " s'il est clair, doux et piquant et qu'il ne soit point suranné ". Quant à la bière, seule la petite bière, dite de ménage, peut être autorisée car, si elle est grosse, épaisse et trouble, faite d'une eau boueuse et impure et que l'on y ait mis des drogues chaudes, pleines de sel, " comme on le fait ordinairement en Angleterre, en Hollande et en Allemagne » il n'est pas douteux qu'elle prédispose franchement à la gravelle et qu'elle doit être énergiquement prohibée. Sur ce point Venette est formel.

Il est de même " des pastez de haut goût, de tourtes, de bisques, de gasteaux, de champignons, de trouffes, de jambons salez, de fromages raffinez, de ragouts épicés et d'autres friandises qui servent de matière aux pierres, quand on en abuse ". Quant aux coquillages et en particulier aux huitres qui, dit Venette « entre tous les coquillages sont les meilleurs », ils ne prédisposent nullement à la gravelle ainsi que le prouve l'expérience, puisque les autochtones qui en consomment beaucoup, ne sont presque jamais atteints de cette maladie. D'ailleurs, l'auteur pense que " la disette du sel est la cause que les Nations du Septentrion sont beaucoup plus sujettes à la pierre que celles du Midy qui n'en manquent pas et où tous les aliments sont salés : pain, chair, herbes et racines ".

Venette célèbre naturellement les vertus classiques au point de vue de la diurèse de " l'oignon blanc pilé et trempé dans un verre de vin blanc " et aussi " des poireaux qui ont une belle vertu pour chasser hors de notre corps par les urines, les pierres, les sablons et les glaires, qu'il n'y a point de remède qui pénètre plus, qui atténue d'avantage et qui excite plus les urines que ceux-cy ". Précurseur de son concitoyen Bridault qui devait vanter au siècle suivant les qualités apéritives, diurétiques et emménagogues de la carotte, Venette préconise, après Van Helmont, l'usage de ce légume dans le régime des lithiasiques qui se trouveront bien également de l'emploi du fenouil déjà recommandé dans l'Antiquité par Dioscoride et au $16^{\mathrm{e}}$ siècle par Rondelet.

Enfin, le chapitre de la phytothérapie se termine par 
l'énumération de nombreux diurétiques froids qui paraissent d'une utilité plus ou moins contestable mais qui néanmoins peuvent contribuer véritablement à la cure de diurèse si profitable aux graveleux.

La thérapeutique proprement dite de Venette comprend d'abord toute une série de petits soins qui ne sont pas négligeables, tels que les lavements, les vomitifs, les purgations qui devront être « pratiquées par précaution 4 ou 6 fois l'année pendant le printemps ou l'automne ". On se félicitera chez ces malades de la présence d'hémorroïdes dont le flux sera une excellente dérivation naturelle et on devra, à l'instar d' Hippocrate, " saigner des parties inférieures les jeunes gens sujets à la pierre ". Le bain tiède dont on ne devra pas abuser cependant pour ne pas trop déprimer le patient, rend d'immenses services car, ainsi que le dit fort bien Venette : "il dilatera les vaisseaux internes et les disposera à laisser doucement couler par leurs cavités les sablons et les sécrétions glaireuses ». Et chacun sait, médecins ou profanes combien est encore utile cette pratique simple et inoffensive qui apporte tant de soulagement aux patients en proie aux coliques néphrétiques. Ainsi tous ces préceptes thérapeutiques sont parfaitement judicieux et marqués au coin du plus parfait bon sens.

Si Venette vante exagérément les vertus lithotriptiques du sang de lièvre, qui est, d'après lui, « l'animal le plus propre à détruire les semences de pierre que nous avons parmy nostre sang ", sacrifiant ainsi à la mode des produits opothérapiques que Pline déjà appelait " les remèdes vivants ", Venette se borne à prescrire une thérapeutique très discrète et prudente. II n'ordonne ni la poudre d'yeux d'écrevisses, ni le bézoard «cette pierre ainsi dénommée par les Allemands et qui vient dans le grand coin des yeux des cerfs ", ni cette fameuse pierre néphrétique provenant des bords de la rivière des Amazones, dont « les Sauvages et les Espagnols disent tant de bien et que nous avons vue si inefficace pour soulager les affres ". II ne prescrit pas davantage le corail ni les coquilles d'œufs, car, remarque-t-il, avec raison, « que peut-on espérer de favorable de toutes les pierres pour détruire celles qui s'engendrent dans nos corps ? " et le clinicien de conclure sagement : " En vérité, on n'a pas assez examiné toutes ces matières et l'on donne souvent à yeux clos sur des remèdes, qui, au lieu d'avoir quelques vertus bienfaisantes, en ont non seulement l'inutilité, mais de malfaisantes et fâcheuses. On doit donc les retrancher de nos compositions que l'on nomme lithotriptiques, qui dans cela seront encore assez composées et auront assez de vertu ". Voilà donc un thérapeute qui n'est pas partisan de la polypharmacie. N'est-ce point là une grande vertu en ce 17 e siècle où les mélanges les plus hétéroclites, telle la thériaque, étaient préparés avec plus d'aveugle conviction que de raison et d'efficacité chez les apothicaires.

Pratiquement, il ne prescrit guère que la térébenthine et encore, dit-il, il « faut n'en user que deux ou trois fois la semaine " pendant quatre ou cinq mois seulement et plutôt en hiver qu'en été. Il l'administre sous forme de pilules à jeun une heure avant le petit déjeuner. Ce remède qu'il utilise depuis 20 ans, est efficace à la fois contre la goutte et la gravelle. Ce n'est qu'accessoirement qu'il ordonne aussi quelques gouttes du baume de la Mecque et du baume du Pérou. Or, à la fin du 19 e siècle, les meilleurs auteurs comme Trousseau et Pidoux, ne formulaient pas d'autre traitement à leurs graveleux âgés, atteints de catarrhe chronique de la vessie, car l'essence de térébenthine, comme le dit Mauquat, calme les douleurs, tarit la production du pus et rétablit l'acidité des urines. Et, s'il est vrai qu'elle est contre indiquée dans les affections aiguës du rein, Bouchard lui-même recommandait constamment la térébenthine comme dissolvant des calculs biliaires qui sont fondus par le très classique remède de Durante.

Certes, depuis le début du $20^{\mathrm{e}}$ siècle, la thérapeutique de la lithiase rénale, s'est enrichie de nombreux solvants de l'acide urique et cette affection a été démembrée en trois groupes suivant la constitution chimique des calculs : phosphatiques, oxaliques et surtout uriques, les plus nombreux et les plus fréquents. Mais il n'en reste pas moins vrai que pendant deux siècles, le traitement fondamental diététique et pharmacologique de la gravelle est demeuré celui que formulait en 1701 le livre posthume du doyen du Collège des Médecins de la Rochelle. $\mathrm{Si}$, au $18^{e}$ siècle, Morgagni a donné une bonne description des lithiases rénale et biliaire, si Van Swieten en a précisé l'étiologie, si enfin, Boerhaave - nommé en 1701 professeur de médecine théorique à l' Université de Leyde, l'année même de la publication du « Traité des Pierres » de Venette - a édicté les principes de la diététique des graveleux, n'oublions pas que le médecin rochelais du $17^{e}$ siècle peut à bon droit revendiquer sur ces trois médecins étrangers du $18^{e}$ siècle, une très légitime et incontestable priorité.

\section{REFERENCES}

1. BARRAUD C. : Nicolas Venette et son traité "Des pierres ». Mémoires de la Société Française d'Histoire de la Médecine et de ses Filiales. 1951, IV, 13-18.

2. COLINA. : Dictionnaire des noms illustres en médecine. Bruxelles, Prodim, 1994 : 149.

3. DUPONT M. : Dictionnaire historique des médecins. Paris, Larousse-Bordas, $1999: 563$.

4. PADOUE XV-XVIII siècles. Les siècles d'or de la médecine. 
Milano, Electa, $1989: 17$

5. PASTEUR C.L. : Les femmes et les médecins. Paris, Zulma, $1997: 19-25$.

6. PATIN C. : Lyceum Patavinum sive icones et vitae professorum, Patavii 1682 publice docentium. Frambotti, Padova, 1682.

7. PORTER R. : Spreading carnal knowledge or selling dirt cheap? Nicolas Venette's Tableau de l'amour conjugal in eighteenth century England. J. Eur. Stud., 1984, 14/4 : 233-255.

8. RUSH J. : The role of the eunuch and the hermaphrodite in : Nicolas Venette's Tableau de l'amour Considéré dans l'estat du Mariage. J. Eur. Stud., 2004, 34/3 : 195-214.

9. VENETTE N. : La Génération de l'homme, ou Tableau de l'amour conjugal. Nouvelle édition, revue et augmentée par le Dr Caufeynon. Paris, Jean Fort, 1907.

10. ZWANG. G.: Histoire des peines de sexe. Paris, Maloine, 1994 : 194-204.

Manuscrit reçu : février 2006 ; accepté février 2006.

ABSTRACT

Nicolas Venette (1633-1698): first French sexologist and pioneer in the field of urinary calculi

\section{Georges ANDROUTSOS}

Nicolas Venette (Charles Patin) was a great physician of the $17^{\text {th }}$ century. He left works on scurvy, mineral waters, urinary calculi, sexology, etc. His master works were " $L a$ Génération de l'homme ou Tableau de l'amour conjugal' and "Traité des pierres". He is considered to be the first French sexologist and a leading pioneer in the field of urinary calculi.

Key words: Venette, pioneer, sexology, urinary calculi 